\title{
The Magnetite nanoparticles in theranostic applications
}

\begin{abstract}
Currently, nanotechnologies are widely used in biomedicine, science and industry. It is known that the application of magnetite nanoparticles as nanostructured carriers is one of the promising areas of biomedicine. The formation of polyelectrolyte or nanocomposite microcapsules is widely applied for the coating of magnetic nanoparticles. The microcapsules containing magnetite nanoparticles have good perspectives for theranostics applications, but careful study of their biodistribution and toxicity is necessary to prove the safety of these structures as a diagnostic and therapeutic agents.
\end{abstract}

Keywords: Magnetite nanoparticles, Theranostics, MRI contast agents, Targeted drug delivery
Volume 5 Issue 3 - 2017

\author{
Olga S Godage,' Alla B Bucharskaya,' Nikita \\ A Navolokin,' Galina N Maslyakova,' Sergey \\ V German, ${ }^{2}$ Dmitry A Gorin ${ }^{2}$ \\ 'Saratov State Medical University, Russia \\ ${ }^{2}$ Saratov State University, Russia
}

Correspondence: Alla B Bucharskaya, Saratov State Medical University, Saratov, Russia, Email allaalla_72@mail.ru

Received: March 23, 2017 | Published: April 20, 2017

\section{Overview}

Recently, more and more research in the field of nanotechnology is aimed to solve the problems of practical medicine. A lot of works is devoted to applications of magnetite nanoparticles in biomedicine, for example, magnetite nanoparticles can be used to enhance contrast and improve diagnostic sensitivity in (MRI) ${ }^{1-3}$ targeted delivery ${ }^{4-6}$ hyperthermia. ${ }^{7,8}$ tissue engineering. ${ }^{9}$ Magnetite is quite a promising material for biomedical applications, in different studies indicates that such nanoparticles have low toxicity, good biocompatibility and sufficient magnitude of effective magnetic characteristics. ${ }^{10,11}$ Low toxicity was also confirmed by vivo studies and morphological examination of internal organs of animals after different methods of administration of magnetite nanoparticles.$^{12-14}$

Stability of nanoparticles is the most important characteristic for the biomedical applications. Surface modification of nanoparticles enables them to ensure not only colloidal stability but also biocompatibility. The aggregation and oxidation were observed for inorganic nanoparticles. Coatings of organic and inorganic nature for surface modification of nanoparticles are used to prevent actions like aggregation, sedimentation. ${ }^{15,16}$ Gold modifies the surface in such a way that increases the stability of nanoparticles in aqueous solution ${ }^{17,18}$ Dextran and its derivatives have a high biocompatibility and are used for stabilization of nanoparticles. ${ }^{19,20}$ Silicon dioxide is often used for stabilization of inorganic nanoparticles because one prevents the aggregation, oxidation of the nanoparticles and as results provides high stability of the colloid. ${ }^{21}$ and reduces toxicity. ${ }^{22}$ The size and charge of nanoparticles is of great importance for biomedical applications. The larger the particle, the shorter its half-life in plasma, the higher the charge of the nanoparticles, the less time they remain the bloodstream..$^{23}$ The nanoparticle absorption from the bloodstream depends on their size..$^{24}$

The particle chemical composition, its size and shape should be considered for successful use of a magnetic field on the nanoparticles, since these parameters determine the magnetic properties of the nanoparticles. The size of the nanoparticles determines the speed of excretion, and also their distribution in the body ${ }^{25}$ based on this the drug delivery to certain organs and tissues is possible. Such type of targeted delivery of drugs allows to reduce the medicine dosage on $20 \%$ of the systemic dose and does not affect healthy tissue, thereby the occurrence of side effects can be avoided. In particular, this is an important during chemotherapy of cancer patients, in which healthy organs and tissues are affected due to undesired biodistribution and high toxicity. ${ }^{26-28}$

At the present time the new trend is developing in medicine which is called theranostics and aims at the same time to the therapy and diagnostics of diseases. Multifunctional nanostructured carriers are developed which find wide application in enzymatic catalysis, controlled release, and directed drug delivery in medicine. Magnetic nanoparticles consistent with this new challenge entirely, as the possibility of their use exists for both diagnosis and treatment. ${ }^{29}$ Magnetic nanoparticles may be used in theranostics to enhance contrast and improve diagnostic sensitivity of MRI as it was stated above. ${ }^{1-3}$ as well as for targeted delivery of drugs and implementation of hyperthermia. ${ }^{4-6}$

Magnetite nanoparticles are used for fabrication of magnitolyposomes or microcapsules..$^{30-34}$ Microencapsulation is the process of surrounding or enveloping one substance within another substance on a very small scale. The capsule size can varied from less than one micron to several hundred microns in size. ${ }^{35}$ The microcapsules can contain various substances sush as nanoparticles, drugs, contrast agents. Microcapsules have small size, large internal volume, large enough surface, have a high effective load of active substances and the flexibility of the physico-chemical properties and are used as micro and sub micro carriers for the development of new effective forms of drugs with prolonged and/or controlled release of the bioactive components (proteins, including enzymes, peptides, hormones, various antigens, DNA, RNA, etc. It can be used as delivery systems for anticancer and antimicrobial drugs, as well as in gene therapy, with the ability to vary the size, structure and physicochemical properties of the microcapsules . ${ }^{36-38}$ Many research works demonstrate that, as a rule, the capsules are well tolerated by living systems in vivo and in vitro. ${ }^{39,40}$

\section{Conclusion}

Thus, at present time nanotechnology occupies an important place among the innovative methods of diagnostics and treatment. One of the rapidly developing areas is associated with the creation of effective nanosystems aimed at theranostics - to the therapy and diagnostics of diseases at the same time. The microcapsules containing magnetite nanoparticles have good perspectives for theranostics applications, but careful study of their biodistribution and toxicity is necessary to 
prove the safety of these structures as a diagnostics and therapeutic agents.

\section{Acknowledgements}

The study was supported by the Government of the Russian Federation (grant №14.Z50.31.0004 to support scientific research projects implemented under the supervision of leading scientists at Russian institutions and Russian institutions of higher education).

\section{Conflict of interest}

None.

\section{References}

1. Stephen ZR, Kievit FM, Zhang M Magnetite nanoparticles for medical MR Imaging. Mater Today (Kidlington). 2011;14(7-8):330-338.

2. Pankhurst QA, Connolly J, Jones SK et al. Applications of magnetic nanoparticles in biomedicine. Journal Physics D: Applied Physics. 2003;36(13):167-181.

3. Joan Estelrich, María Jesús Sánchez-Martín, Maria Antònia Busquets Nanoparticles in magnetic resonance imaging: from simple to dual contrast agents. Int J Nanomedicine. 2015;10:1727-1741.

4. Johannsen M, Gneveckow U, Thiesen B et al. Thermotherapy of prostate cancer using magnetic nanoparticles: Feasibility, imaging, and threedimensional temperature distribution. Eur Urol. 2007;52(6):1653-1661.

5. Ito A, Tanaka $\mathrm{K}$, Honda $\mathrm{H}$ et al. Complete Regression of Mouse Mammary Carcinoma with a Size Greater than $15 \mathrm{~mm}$ by Frequent Repeated Hyperthermia Using Magnetite Nanoparticles J Biosci Bioeng. 2003;96(4):364-369.

6. Ito A, Shinkai M, Honda H, Kobayashi $\mathrm{T}$ Medical application of functionalized magnetic nanoparticles. $J$ Biosci Bioeng. 2005;100(1):1-11.

7. R Jurgons, C Seliger, A Hilpert et al. Drug loaded magnetic nanoparticles for cancer therapy. Journal of Physics: Condensed Matter. 2006;18(38):2893-2902

8. Lübbe AS, Alexiou C, Bergemann C Clinical applications of magnetic drug targeting. J Surg Res. 2001;95(2):200-206.

9. Michael D Kaminski, Axel J Rosengart Detoxification of blood using injectable magnetic nanoparticles: a conceptual technology description. Journal of Magnetism and Magnetic Materials. 2005;293(1):389-403.

10. Prodan AM, Iconaru SL, Ciobanu CS et al. Iron oxide magnetic nanoparticles: characterization and toxicity evaluation by in vitro and in vivo assays. Journal of Nanomaterials. 2013:1-10.

11. Pershin AG, Sazonov AE, Milto IV The use of magnetic nanoparticles in biomedicine. Bulletin of the Siberian medicine. 2008;2:70-78.

12. Navolokin NA, GN Maslyakova, AB Bucharskya et al. Morphological changes in the kidney, liver and spleen during prolonged administration of iron nanoparticles. Journal of Physics: Conference Series. 2012;345:012043

13. ilto IV, Mikhailov GA, Ratkin AV et al. Influence of nanoparticles on the morphology of internal organs of mice with intravenous solution $\mathrm{Fe} 3 \mathrm{O} 4$ nanopowders. Bulletin of the Siberian medicine. 2008;1:32-36.

14. German SV, Inozemtseva OA, Navolokin NA et al. Synthesis of magnetite hydrosols and assessment of their impact on living systems at the cellular and tissue levels using MRI and morphological investigation. Nanotechnologies in Russia. 2013;8(7):573-580.

15. Massia SP, Stark J, Letbetter D S Surface immobilized dextran limits cell adhesion and spreading. Biomaterials. 2000;21(22):2253-2261.
16. Yu J, Lee CW, Im SS, Lee JS Structure and magnetic properties of $\mathrm{SiO} 2$ coated $\mathrm{Fe} 2 \mathrm{O} 3$ nanoparticles synthesized by chemical vapor condensation process. CiteSeerX10M. 2003;4:55-59.

17. Chen M, Yamamuro S, Farrell D et al. Gold-coated iron nanoparticles for biomedical applications. Journal Applied Physics. 2003;93(10):7551-7553.

18. Weilie Zhou, A Kumbhar, J Wiemann et al. Gold-coated iron (Fe@Au) nanoparticles: synthesis, characterization, and magnetic field-induced self-assembly. Journal Solid State Chemistry. 2001;159:26-31.

19. Lacava LM, Lacava ZG, MF Da Silva et al. Magnetic resonance of a dextran coated magnetic fluid intravenously administered in mice. Journal Biophys. 2001;80(5):2483-2486.

20. Berry CC, Wells S, Charles S et al. Dextran and albumin derivatised iron oxide nanoparticles: influence on fibroblasts in vitro. Biomaterials. 2003;24(25):4551-4557.

21. P Mulvaney, LM Liz-Marzán, M Giersigc, T Ung Silica encapsulation of quantum dots and metal clusters. Journal of Material Chemistry. 2000;10:1259-1270.

22. Lesnikovich AE, Shunkevich TM, Naumenko VN et al. II Dispersity of magnetite in magnetic liquids and the interaction with a surfactant. $J$ Magn Mater. 1990;85:14-16.

23. Chouly C, Pouliquen D, Lucet I et al. Development of superparamagnetic nanoparticles for MRI: effect of particle size, charge and surface nature on biodistribution. J Microencapsul. 1996;13(3):245-255.

24. Tarakhovsky YS Intelligent lipid nanocontainers in targeted delivery of drugs. Biochemistry (Mosc). 2011;75(7):811-824.

25. Soo Jin Choia, Jin Ho Choy Effect of physico-chemical parameters on the toxicity of inorganic nanoparticles. Journal Materials Chemistry. 2011;21:5547-5554.

26. Laurent S, Forge D, Port M et al. Magnetic iron oxide nanoparticles: synthesis, stabilization, vectorization, physicochemical characterisations, and biological applications. Chemical Reviews. 2008;108(6):2064-2110.

27. Jordan A, Scholz R, Maier Hauff K et al. Presentation of a new magnetic field therapy system for the treatment of human solid tumors with magnetic fluid hyperthermia. Journal of Magnetism and Magnetic Materials. 2001;225(1-2):118-126.

28. Hingorani DV, Bernstein AS, Pagel MD A review of responsive MRI contrast agents: 2005-2014. Contrast Media Mol Imaging. 2015;10(4):245-265.

29. Gobbo OL, Sjaastad K, Radomski MW et al. Magnetic Nanoparticles in Cancer Theranostics. Theranostics. 2015;5(11):1249-1263.

30. German SV, Navolokin NA, Kuznetsova NR et al. Liposomes loaded with hydrophilic magnetite nanoparticles: preparation and application as contrast agents for magnetic resonance imaging. Colloids and Surfaces B: Biointerfaces. 2015;135:109-115.

31. Lacava ZGM, Garcia VAP, Lacava LM Biodistribution and biocompalibility investigation in magnitoliposome treated mice. Spectroscopy. 2009;18(4):597-603.

32. Al-Jamal WT, Kostarelos K Liposome-nanoparticle hybrids for multimodal diagnostic and therapeutic applications. Nanomedicine. 2007;2(1):85-98.

33. Polyak B, Friedman G Magnetic targeting for site-specific drug delivery: applications and clinical potential Expert Opin Drug Deliv. 2009;6(1):53-70.

34. Sukhorukov GB, Rogach AL, Garstka M et al. Multifunctionalized polymer microcapsules: novel tools for biological and pharmacological applications. Small. 2007;3(6):944-955. 
35. Jyothi Sri S, Seethadevi A, Suria Prabha K et al. Microencapsulation: a review international journal of pharma and bio sciences. International Journal of Pharma and Bio Sciences. 2012;3(1):509-531.

36. De Geest B G, Vandenbroucke RE, Guenther AM et al. Intracellularly degradable polyelectrolyte microcapsules. Advaced Materials. 2006;18(8):1005-1009.

37. Zhao Q, Han B, Wang Z et al. Hollow chitosan-alginate multilayer microcapsules as drug delivery vehicle: doxorubicin loading and in vitro and in vivo studies. Nanomedicine. 2007;3(1):63-74.
38. De Koker S, De Geest B G, Cuvelier C et al. In vivo Cellular Uptake, Degradation, and Biocompatibility of Polyelectrolyte Microcapsules. Advanced Function Materials. 2007;17(18):3754 -3763.

39. Javier AM, Kreft O, Semmling $M$ et al. Uptake of colloidal polyelectrolyte-coated particles and polyelectrolyte multilayer capsules by living cells. Advanced Materials. 2008;20(22):4281-4287.

40. Kirchner C, Javier AM, Susha AS et al. Cytotoxicity of nanoparticleloaded polymer capsules. Talanta. 2005;67(3):486-491. 